Supporting Information

\title{
Redox Behavior of Ferrocene-Containing Rotaxane: Transposition of Wheel of Rotaxane by Redox Reaction of Ferrocene Moiety Tethered at the End of Axle
}

\author{
Nobuhiro Kihara, ${ }^{*}$ Makiko Hashimoto, and Toshikazu Takata ${ }^{\dagger} *$ \\ Department of Applied Chemistry, Graduate School of Engineering, Osaka Prefecture \\ University, Gakuen-cho, Sakai, Osaka 599-8531, Japan \\ ${ }^{\ddagger}$ Present address: Department of Organic and Polymeric Materials, Tokyo Institute of \\ Technology, Ookayama, Meguro, Tokyo 152-8552, Japan \\ Tel: $+81-722-54-9295$ \\ Fax: +81-722-54-9910 \\ e-mail: kihara@chem.osakafu-u.ac.jp
}

$\underline{\text { Experimental }}$

Methods and Materials. ${ }^{1} \mathrm{H}$ and ${ }^{13} \mathrm{C}$ NMR spectra were recorded on a JEOL JNM-GX-270 $(500 \mathrm{MHz})$ spectrometer using tetramethylsilane as an internal standard. IR spectra were recorded on a JASCO FT/IR-230 spectrometer. UV-vis spectra were recorded on a JASCO V-560 spectrometer. FAB-MS analysis were made on a FINNIGAN MAT TSQ-70 instrument. Melting points were measured on a Yanako MP-3 instrument. Preparative GPC was carried out using JAI LC-908 equipped with JAIGEL-1C and JAIGEL-2C columns. Cyclic voltammograms were recorded on a Hokuto Denko HSV-100 instrument with $\mathrm{Ag} / \mathrm{AgNO}_{3}$ reference electrode, glassy carbon working electrode, and platinum wire counter electrode at $298 \mathrm{~K}$ in $0.1 \mathrm{~mol} / \mathrm{L}$ tetrabutylammonium perchlorate or hexafluorophophate in acetonitrile. Ammonium salt $\mathbf{2}^{1}$ and ferrocenecarboxylic anhydride ${ }^{2}$ were prepared according to the literatures. Acetonitrile, $N, N$-dimethylformamide, and triethylamine were used after distillation over calcium hydride. Dichloromethane was dried with phosphorous pentoxide followed by distillation over calcium hydride. Other chemicals were reagent grade and used without further purification.

${ }^{1}$ Kawasaki, H.; Kihara, N.; Takata, T. Chem. Lett., 1999, 1015.

${ }^{2}$ Rambacher, P.; Mäke, S. Angew. Chem. Int. Ed. Engl., 1968, 7, 465. 
Preparation of ammonium salt $\mathbf{2 c}$ and $\mathbf{2 d}$

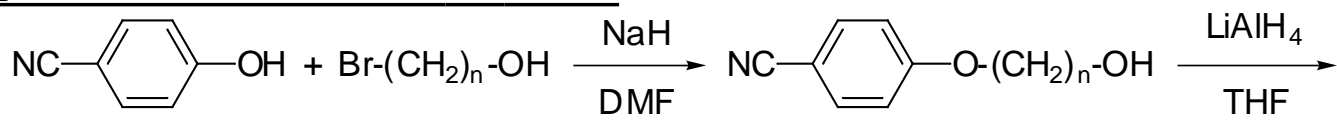

5

6

7

$\mathrm{O}-\left(\mathrm{CH}_{2}\right)_{\mathrm{n}-\mathrm{OH}}$

8

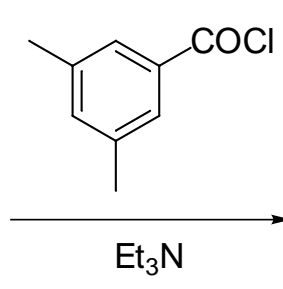

$\mathrm{Et}_{3} \mathrm{~N}$<smiles>Cc1cc(C)cc(C[NH2+]Cc2ccc(OCC(O)C(F)(F)F)cc2)c1</smiles>

9<smiles>Cc1cc(C)cc(C(=O)NCc2ccc(OCCO)cc2)c1</smiles>

\section{Hydroxynitrile 7}

After a mixture of sodium hydride (3.10 $\mathrm{g}$ of $60 \%$ dispersion in mineral oil, $77.5 \mathrm{mmol}$ ) and 4-cyanophenol (3.62 g, $30.2 \mathrm{mmol})$ in DMF (50 mL) was stirred at $50{ }^{\circ} \mathrm{C}$ for $2 \mathrm{~h}$, 2-bromoethanol (3.78 g, $27.6 \mathrm{mmol})$ was added, and the reaction mixture was allowed to stand at $100{ }^{\circ} \mathrm{C}$ for $16 \mathrm{~h}$. After water $(100 \mathrm{~mL})$ was added, the reaction mixture was extracted by ethyl acetate. The organic layer was washed by water (twice), dried over magnesium sulfate, and evaporated in vacuo. The crude product $(2.82 \mathrm{~g}, 17.3 \mathrm{mmol}, 63 \%)$ was analytically pure, and was used without further purification.

7c: ${ }^{1} \mathrm{H}-\mathrm{NMR}\left(\mathrm{CDCl}_{3}, 270 \mathrm{MHz}\right) \delta 7.59(\mathrm{~d}, J=8.9 \mathrm{~Hz}, 2 \mathrm{H}, \mathrm{Ar}-\mathrm{H}), 6.98(\mathrm{~d}, J=8.9 \mathrm{~Hz}, 2 \mathrm{H}$, Ar-H), $4.16\left(\mathrm{t}, J=6.4 \mathrm{~Hz}, 2 \mathrm{H}, \mathrm{CH}_{2}\right), 4.00\left(\mathrm{t}, J=6.4 \mathrm{~Hz}, 2 \mathrm{H}, \mathrm{CH}_{2}\right) \mathrm{ppm}$.

7d: ${ }^{1} \mathrm{H}-\mathrm{NMR}\left(\mathrm{CDCl}_{3}, 270 \mathrm{MHz}\right) \delta 7.57(\mathrm{~d}, J=9.2 \mathrm{~Hz}, 2 \mathrm{H}, \mathrm{Ar}-\mathrm{H}), 6.93(\mathrm{~d}, J=9.2 \mathrm{~Hz}, 2 \mathrm{H}$, Ar-H), 4.00 (t, $\left.J=6.5 \mathrm{~Hz}, 2 \mathrm{H}, \mathrm{OCH}_{2}\right), 3.66$ (t, $\left.J=6.5 \mathrm{~Hz}, 2 \mathrm{H}, \mathrm{HO}-\mathrm{CH}_{2}\right), 1.85-1.00(\mathrm{~m}, 20 \mathrm{H}$, $\left.\mathrm{CH}_{2}\right)$ ppm.

\section{Amonoalcohol 8}

To a suspension of lithium almunum hydride $(3.51 \mathrm{~g}, 92.5 \mathrm{mmol})$ in THF (35 mL) was added dropwise a solution of alcohol 7c $(2.82 \mathrm{~g}, 17.3 \mathrm{mmol})$ in THF $(65 \mathrm{~mL})$ at $0{ }^{\circ} \mathrm{C}$ under an argon atmosphere. After $1.5 \mathrm{~h}$, saturated sodium sulfate solution was added dropwise until the evolution of hydrogen ceased. The precipitate was filtrated off, and the filtrate was diluted by ethyl acetate, washed with water (twice), dried over magnesium sulfate, and evaporated in vacuo. The crude product was chromatographed (silica gel, eluent: chloroformmethanol, 4/1 v/v) to give $932 \mathrm{mg}(5.58 \mathrm{mmol}, 32 \%)$ of aminoalcohol $8 \mathrm{c}$ as a yellow-brown 
powder.

8c: ${ }^{1} \mathrm{H}-\mathrm{NMR}\left(\mathrm{CDCl}_{3}, 270 \mathrm{MHz}\right) \delta 7.22(\mathrm{~d}, J=8.6 \mathrm{~Hz}, 2 \mathrm{H}, \mathrm{Ar}-\mathrm{H}), 6.85(\mathrm{~d}, J=8.6 \mathrm{~Hz}, 2 \mathrm{H}$, Ar-H), 4.07 (t, $\left.J=6.4 \mathrm{~Hz}, 2 \mathrm{H}, \mathrm{CH}_{2}\right), 3.93\left(\mathrm{t}, J=6.4 \mathrm{~Hz}, 2 \mathrm{H}, \mathrm{CH}_{2}\right), 3.80\left(\mathrm{~s}, 2 \mathrm{H}, \mathrm{N}-\mathrm{CH}_{2}-\mathrm{Ar}\right)$ ppm.

8d: ${ }^{1} \mathrm{H}-\mathrm{NMR}\left(\mathrm{CDCl}_{3}, 270 \mathrm{MHz}\right) \delta 7.23(\mathrm{~d}, J=8.9 \mathrm{~Hz}, 2 \mathrm{H}, \mathrm{Ar}-\mathrm{H}), 6.86(\mathrm{~d}, J=8.9 \mathrm{~Hz}, 2 \mathrm{H}$, Ar-H), $3.94\left(\mathrm{t}, J=5.4 \mathrm{~Hz}, 2 \mathrm{H}, \mathrm{OCH}_{2}\right), 3.80\left(\mathrm{~s}, 2 \mathrm{H}, \mathrm{N}-\mathrm{CH}_{2}-\mathrm{Ar}\right), 3.63(\mathrm{t}, J=5.4 \mathrm{~Hz}, 2 \mathrm{H}$, $\left.\mathrm{HO}-\mathrm{CH}_{2}\right), 1.74-1.30\left(\mathrm{~m}, 20 \mathrm{H}, \mathrm{CH}_{2}\right) \mathrm{ppm}$.

\section{Amide 9}

To a solution of $8 \mathbf{c}(932 \mathrm{mg}, 5.58 \mathrm{mmol})$ in chloroform $(7.0 \mathrm{~mL})$ were added triethylamine (1.00 mL, $7.12 \mathrm{mmol})$ and a solution of 3,5-dimethylbenzoyl chloride (940 mg, $5.58 \mathrm{mmol}$ ) in chloroform $(1.0 \mathrm{~mL})$. After $24 \mathrm{~h}$, the reaction mixture was washed with water then brine, dried over magnesium sulfate, and evaporated in vacuo to give $1.57 \mathrm{~g}(5.25 \mathrm{mmol}, 94 \%)$ of amide $9 \mathbf{c}$ as a yellow solid. The crude product was used without further purification.

9c: ${ }^{1} \mathrm{H}-\mathrm{NMR}\left(\mathrm{CDCl}_{3}, 270 \mathrm{MHz}\right) \delta 7.37$ (s, 2H, Ar-H), 7.27 (d, $\left.J=8.9 \mathrm{~Hz}, 2 \mathrm{H}, \mathrm{Ar}-\mathrm{H}\right), 6.92$ (d, $J=8.9 \mathrm{~Hz}, 2 \mathrm{H}, \mathrm{Ar}-\mathrm{H}), 3.94$ (t, $J=6.4 \mathrm{~Hz}, 2 \mathrm{H}, \mathrm{CH}_{2}$ ), 4.65 (d, $J=5.7 \mathrm{~Hz}, 2 \mathrm{H}, \mathrm{N}-\mathrm{CH}_{2}-\mathrm{Ar}$ ), $4.08\left(\mathrm{~d}, J=5.7 \mathrm{~Hz}, 2 \mathrm{H}, \mathrm{CH}_{2}\right), 4.00-3.96\left(\mathrm{~m}, 2 \mathrm{H}, \mathrm{HO}-\mathrm{CH}_{2}\right) 2.34\left(\mathrm{~s}, 6 \mathrm{H}, \mathrm{Ar}-\mathrm{CH}_{3}\right) \mathrm{ppm}$.

9d: ${ }^{1} \mathrm{H}-\mathrm{NMR}\left(\mathrm{CDCl}_{3}, 270 \mathrm{MHz}\right) \delta 7.37(\mathrm{~s}, 2 \mathrm{H}, \mathrm{Ar}-\mathrm{H}), 7.26(\mathrm{~d}, J=8.6 \mathrm{~Hz}, 2 \mathrm{H}, \mathrm{Ar}-\mathrm{H}), 6.87$ (d, $J=8.6 \mathrm{~Hz}, 2 \mathrm{H}, \mathrm{Ar}-\mathrm{H}), 3.94\left(\mathrm{t}, J=6.4 \mathrm{~Hz}, 2 \mathrm{H}, \mathrm{CH}_{2}\right), 6.30-6.20(\mathrm{br}, 1 \mathrm{H}, \mathrm{NH}), 4.56$ (d, $J=$ $\left.5.4 \mathrm{~Hz}, 2 \mathrm{H}, \mathrm{N}-\mathrm{CH}_{2}-\mathrm{Ar}\right), 3.94\left(\mathrm{t}, J=5.4 \mathrm{~Hz}, 2 \mathrm{H}, \mathrm{OCH}_{2}\right), 3.63\left(\mathrm{q}, J=5.4 \mathrm{~Hz}, 2 \mathrm{H}, \mathrm{HO}-\mathrm{CH}_{2}\right)$, $2.34\left(\mathrm{~s}, 6 \mathrm{H}, \mathrm{Ar}-\mathrm{CH}_{3}\right), 1.58-1.20\left(\mathrm{~m}, 20 \mathrm{H}, \mathrm{CH}_{2}\right) \mathrm{ppm}$.

\section{Ammonium salt 2}

To a suspension of lithium alminum hydride $(1.01 \mathrm{~g}, 26.0 \mathrm{mmol})$ in THF $(30 \mathrm{~mL})$ was added dropwise a solution of amide $9 \mathrm{c}(1.57 \mathrm{~g}, 5.25 \mathrm{mmol})$ in THF $(20 \mathrm{~mL})$ under an argon atmosphere. After the reaction mixture was refluxed for $2 \mathrm{~h}$, saturated sodium sulfate solution was added dropwise until the evolution of hydrogen ceased. The precipitate was filtrated off, and the filtrate was diluted by ethyl acetate, washed with water (twice), dried over magnesium sulfate, and evaporated in vacuo to give $1.60 \mathrm{~g}(5.87 \mathrm{mmol})$ of aminoalcohol as a yellow oil. The product was dissolved in methanol $(4 \mathrm{~mL})$, and the solution was dropwise poured into $3 \%$ aqueous solution of hydrogen hexafluorophosphate $(15 \mathrm{~mL})$. After stirring for $1 \mathrm{~h}$, the white precipitate was isolated by filtration. The crude product was dissolved in chloroformmethanol mixture, and insoluble materials were filtered off. The filtrate was dried over

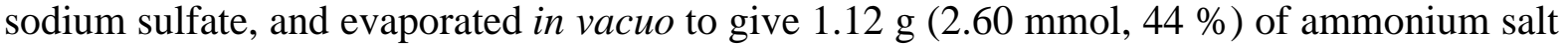
2c as white powder. 2d was prepared in the same manner, and recrystalized from ethyl 
acetate.

2c: ${ }^{1} \mathrm{H}-\mathrm{NMR}\left(\mathrm{CDCl}_{3}, 270 \mathrm{MHz}\right) \delta 7.38(\mathrm{~d}, J=8.9 \mathrm{~Hz}, 2 \mathrm{H}, \mathrm{Ar}-\mathrm{H}), 7.06(\mathrm{~s}, 3 \mathrm{H}, \mathrm{Ar}-\mathrm{H}), 6.97$ $(\mathrm{d}, J=7.6 \mathrm{~Hz}, 2 \mathrm{H}, \mathrm{Ar}-\mathrm{H}), 6.82(\mathrm{~d}, J=7.6 \mathrm{~Hz}, 2 \mathrm{H}, \mathrm{Ar}-\mathrm{H}), 4.65-4.34\left(\mathrm{br}, 2 \mathrm{H}, \mathrm{NH}_{2}\right)$, 4.05-3.88 (m, 4H, $\left.\mathrm{CH}_{2}-\mathrm{N}\right), 3.78-3.71\left(\mathrm{~m}, 2 \mathrm{H}, \mathrm{OCH}_{2}\right), 3.69-3.60\left(\mathrm{~m}, 2 \mathrm{H}, \mathrm{OCH}_{2}\right), 2.30(\mathrm{~s}, 6 \mathrm{H}$, $\left.\mathrm{Ar}-\mathrm{CH}_{3}\right) \mathrm{ppm}$.

2d: ${ }^{1} \mathrm{H}-\mathrm{NMR}\left(\mathrm{CDCl}_{3}, 270 \mathrm{MHz}\right) \delta 7.92(\mathrm{~d}, J=8.6 \mathrm{~Hz}, 2 \mathrm{H}, \mathrm{Ar}-\mathrm{H}), 7.05$ (s, 1H, Ar-H),

6.98 (s, 1H, Ar-H), 6.92 (d, J=8.6 Hz, 2H, Ar-H), 6.82 (d, J = 7.6 Hz, 2H, Ar-H), 4.08-3.98 $\left(\mathrm{m}, 4 \mathrm{H}, \mathrm{NH}_{2}\right), 3.95\left(\mathrm{t}, J=5.4 \mathrm{~Hz}, 2 \mathrm{H}, \mathrm{OCH}_{2}\right), 3.57$ (t, $\left.J=5.4 \mathrm{~Hz}, 2 \mathrm{H}, \mathrm{HO}-\mathrm{CH}_{2}\right), 2.33(\mathrm{~s}, 6 \mathrm{H}$, $\left.\mathrm{Ar}-\mathrm{CH}_{3}\right), 1.82-1.34\left(\mathrm{~m}, 20 \mathrm{H}, \mathrm{CH}_{2}\right)$ ppm.

\section{Preparation of Rotaxane}

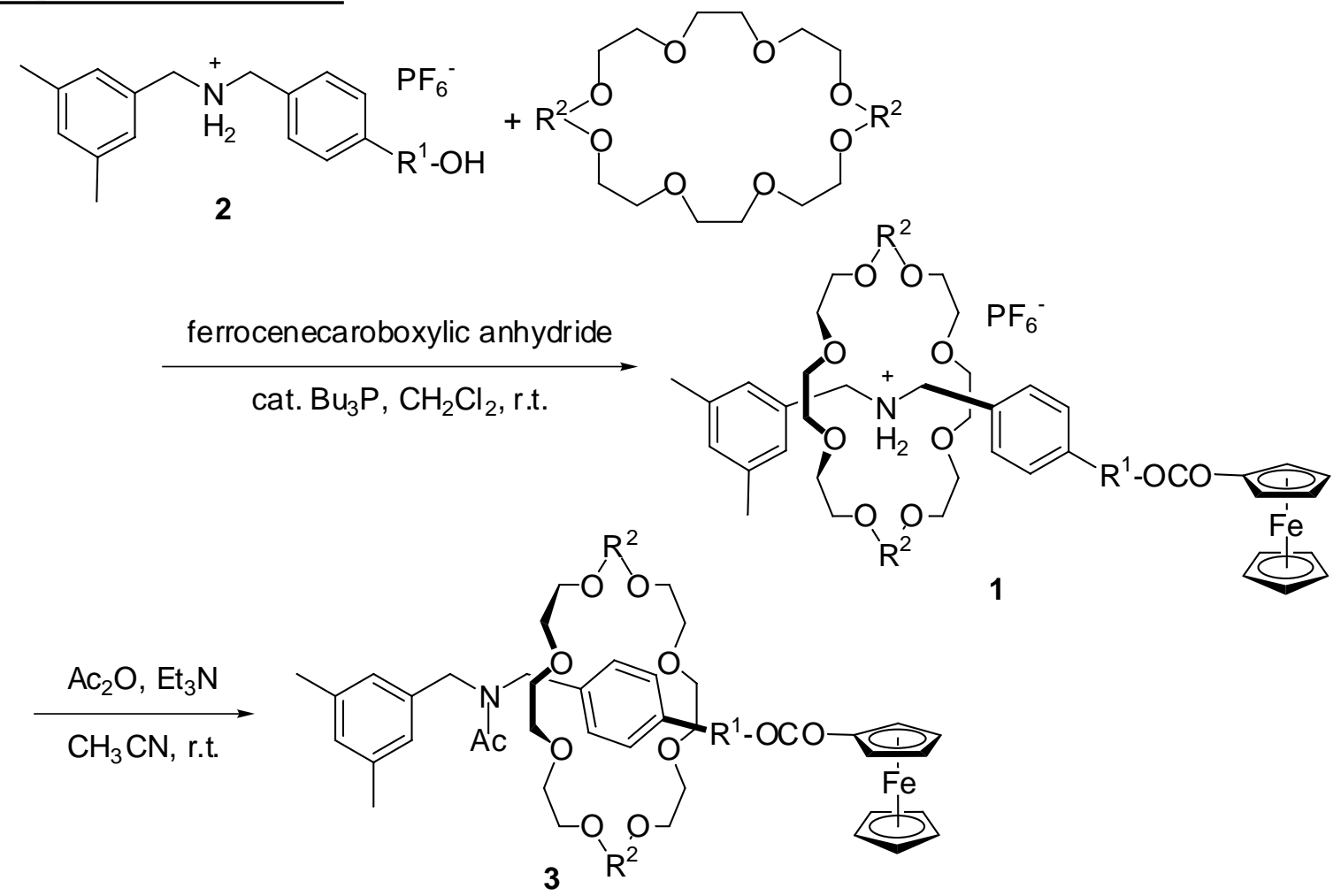

\section{Ammonium-type rotaxane 1: Typical procedure}

To a solution of ammonium salt 2 (42.0 mg, $0.105 \mathrm{mmol})$ and DB24C8 (50.0 mg, 0.112 $\mathrm{mmol})$ in dichloromethane $(0.280 \mathrm{~mL})$ were added ferrocenecarboxylic anhydride $(82.0 \mathrm{mg}$, $0.185 \mathrm{mmol})$ and tributylphosphane $(10.0 \mu \mathrm{L}, 0.0420 \mathrm{mmol})$, and the reaction mixture was allowed to stand at room temperature for $3 \mathrm{~h}$. The reaction mixture was diluted by ethyl acetate, washed by $5 \%$ sodium hydrogen carbonate solution then saturated ammonium hexafuluorophophate solution, dried over magnesium sulfate, and evaporated in vacuo. The 
crude product $(175 \mathrm{mg})$ was purified by preparative GPC to give $103 \mathrm{mg}(0.0529 \mathrm{mmol}$, $91 \%$ ) of rotaxane 1 as a brown crystal. A single crystal was prepared by the slow evaporation of the benzene solution of $\mathbf{1}$.

1a: m.p. $129-135{ }^{\circ} \mathrm{C} .{ }^{1} \mathrm{H}$ NMR $\left(270 \mathrm{MHz} \mathrm{CDCl}_{3}\right) \delta 8.30-7.80\left(2 \mathrm{H}, \mathrm{br}, \mathrm{NH}_{2}\right), 7.39-6.74$ (4H, m, Ar-H), 6.90-6.70 (13H, m, Ar-H), 4.79 (2H, s, Cp), 4.65-4.49 (2H, m, Ar- $\left.\mathrm{CH}_{2}-\mathrm{N}\right)$, 4.48-4.44 (2H, m, Ar- $\left.\mathrm{CH}_{2}-\mathrm{N}\right), 4.43(2 \mathrm{H}, \mathrm{s}, \mathrm{Cp}), 4.10-3.90\left(13 \mathrm{H}, \mathrm{Cp}\right.$ and $\left.\mathrm{CH}_{2}\right), 3.85-3.65(\mathrm{~m}$, $\left.8 \mathrm{H}, \mathrm{CH}_{2}\right), 3.41\left(\mathrm{~s}, 8 \mathrm{H}, \mathrm{CH}_{2}\right), 2.01\left(\mathrm{~s}, 6 \mathrm{H}, \mathrm{CH}_{3}\right)$ ppm. IR $(\mathrm{KBr}) 1708\left(\mathrm{v}_{\mathrm{C}=\mathrm{O}}\right), 840\left(\mathrm{v}_{\mathrm{P}-\mathrm{F}}\right), 557$ $\left(v_{\mathrm{P}-\mathrm{F}}\right) \mathrm{cm}^{-1}$. FAB-MS (m-NBA) $\mathrm{m} / \mathrm{z}=1061[\mathrm{M}]^{+}, 916\left[\mathrm{M}-\mathrm{PF}_{6}\right]^{+}$.

1b: ${ }^{1} \mathrm{H}-\mathrm{NMR}\left(\mathrm{CDCl}_{3}, 270 \mathrm{MHz}\right) \delta$ 7.74-7.64 (br, $\left.\mathrm{NH}_{2}, 2 \mathrm{H}\right), 7.63-7.44(\mathrm{~m}, \mathrm{Ar}-\mathrm{H}, 4 \mathrm{H})$,

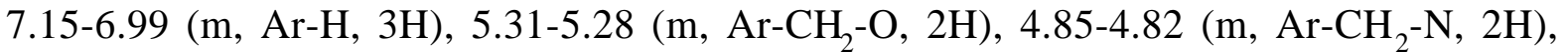
4.66-4.41 (m, Cp and Ar- $\left.\mathrm{CH}_{2}-\mathrm{N}, 6 \mathrm{H}\right), 4.14-4.11$ (s, Cp, 5H), 3.78-3.14 (m, $\left.\mathrm{CH}_{2}, 28 \mathrm{H}\right)$, 2.36-2.34 (m, Ar- $\left.\mathrm{CH}_{3}, 6 \mathrm{H}\right), 1.58-0.97\left(\mathrm{~m}, \mathrm{CH}_{2}, 16 \mathrm{H}\right)$ ppm. IR (KBr) $1708\left(\mathrm{v}_{\mathrm{C}=\mathrm{O}}\right), 840$ $\left(v_{\mathrm{P}-\mathrm{F}}\right), 557\left(\mathrm{v}_{\mathrm{P}-\mathrm{F}}\right) \mathrm{cm}^{-1}$. FAB-MS (m-NBA) $\mathrm{m} / \mathrm{z}=1073[\mathrm{M}]^{+}, 929\left[\mathrm{M}-\mathrm{PF}_{6}\right]^{+}$.

1c: ${ }^{1} \mathrm{H}-\mathrm{NMR}\left(\mathrm{CDCl}_{3}, 270 \mathrm{MHz}\right) \delta$ 7.60-7.40 (br, 2H, $\left.\mathrm{NH}_{2}\right), 7.33-7.28(\mathrm{~m}, 2 \mathrm{H}, \mathrm{Ar}-\mathrm{H})$,

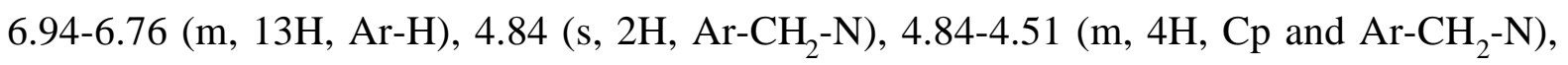
4.43-4.05 (m, 19H, $\mathrm{CH}_{2}$ and $\left.\mathrm{Cp}\right), 3.78-3.70\left(\mathrm{~m}, 8 \mathrm{H}, \mathrm{CH}_{2}\right), 3.41\left(\mathrm{~s}, 8 \mathrm{H}, \mathrm{CH}_{2}\right), 2.14(\mathrm{~s}, 6 \mathrm{H}, \mathrm{Ar}-$ $\left.\mathrm{CH}_{3}\right) \mathrm{ppm}$.

1d: ${ }^{1} \mathrm{H}-\mathrm{NMR}\left(\mathrm{CDCl}_{3}, 270 \mathrm{MHz}\right) \delta$ 7.55-7.40 (br, 2H, $\left.\mathrm{NH}_{2}\right), 7.28-7.23(\mathrm{~m}, 2 \mathrm{H}, \mathrm{Ar}-\mathrm{H})$,

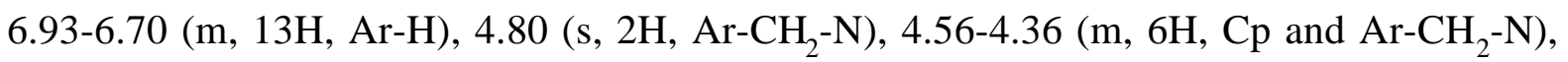
4.23-3.69 (m, 25H, $\mathrm{CH}_{2}$ and $\mathrm{Cp}$ ), 3.49-3.43 (br, 8H, $\mathrm{CH}_{2}$ ), 2.14 (s, 6H, Ar- $\mathrm{CH}_{3}$ ), 1.49-1.22 (m, 20H, $\mathrm{CH}_{2}$ ) ppm.

\section{Nonionic rotaxane 3}

To a solution of rotaxane $1(105 \mathrm{mg}, 0.0986 \mathrm{mmol})$ in acetonitrile $(0.3 \mathrm{~mL})$ were added triethylamine $(50.0 \mu \mathrm{L}, 0.495 \mathrm{mmol})$ and acetic anhydride $(69.6 \mu \mathrm{L}, 0.495 \mathrm{mmol})$, and the reaction mixture was allowed to stand at room temperature for $15 \mathrm{~h}$. The reaction mixture was diluted by chloroform, washed by water then brine, dried over magnesium sulfate, and evaporated in vacuo. The crude product was purified by preparative GPC to give $98.1 \mathrm{mg}$ $(0.0951 \mathrm{mmol}, 96 \%)$ of rotaxane 3 as a brown crystral. A single crystal was prepared by the crystalization in acetonitrile-hexane bilayer system.

3a: ${ }^{1} \mathrm{H}-\mathrm{NMR}\left(\mathrm{CDCl}_{3}, 270 \mathrm{MHz}\right) \delta 8.09$ and 8.01 (two d, Ar-H, 2H, $J=8.1$ and $8.1 \mathrm{~Hz}$ ), 7.18-6.75 (m, Ar-H, 13H), 5.86 and 5.84 (two s, Ar- $\mathrm{CH}_{2}-\mathrm{O}, 2 \mathrm{H}$ ), 5.02-4.95 (m, Cp, 2H), 4.58-4.08 (m, Ar- $\mathrm{CH}_{2}-\mathrm{N}, \mathrm{CH}_{2}$, and $\left.\mathrm{Cp}, 17 \mathrm{H}\right)$, 3.74-3.62 (m, $\left.\mathrm{CH}_{2}, 8 \mathrm{H}\right), 3.40-3.23\left(\mathrm{~m}, \mathrm{CH}_{2}\right.$, 
4H), 2.91-2.83 (m, $\left.\mathrm{CH}_{2}, 4 \mathrm{H}\right), 2.26\left(\mathrm{~s}, \mathrm{CH}_{3}, 6 \mathrm{H}\right), 2.13$ and 2.03 (two s, Ac, 3H) ppm. IR $(\mathrm{KBr}) 1707\left(\mathrm{v}_{\mathrm{C}=\mathrm{O}}\right.$, ester $), 1646\left(\mathrm{v}_{\mathrm{C}=\mathrm{O}}\right.$, amide $) \mathrm{cm}^{-1}$. FAB-MS $(\mathrm{m}-\mathrm{NBA}) \mathrm{m} / \mathrm{z}=970[\mathrm{M}]^{+}, 927$ $[\mathrm{M}-\mathrm{Ac}]^{+}, 812\left[\mathrm{M}-\mathrm{Me}_{2} \mathrm{C}_{6} \mathrm{H}_{3} \mathrm{CH}_{2}\right]^{+}$.

3b: ${ }^{1} \mathrm{H}-\mathrm{NMR}\left(\mathrm{CDCl}_{3}, 270 \mathrm{MHz}\right) \delta$ 8.3-7.97 (m, Ar-H, 2H), 7.25-7.10 (m, Ar-H, 2H), 6.96-6.72 (m, Ar-H, 3H), 5.83-4.68 (m, Ar- $\left.\mathrm{CH}_{2}-\mathrm{O}, 2 \mathrm{H}\right), 5.26-5.14$ (s, Cp, 2H), 4.66-4.23 (m, Ar- $\mathrm{CH}_{2}-\mathrm{N}$ and $\left.\mathrm{Cp}, 11 \mathrm{H}\right), 3.81-2.99$ (m, $\left.\mathrm{CH}_{2}, 28 \mathrm{H}\right), 2.34-2.19$ (m, Ar- $\left.\mathrm{CH}_{3}, 6 \mathrm{H}\right), 1.92-1.21$

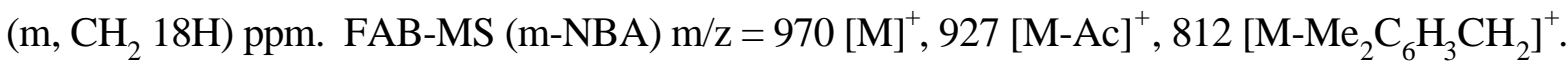
3c: ${ }^{1} \mathrm{H}-\mathrm{NMR}\left(\mathrm{CDCl}_{3}, 270 \mathrm{MHz}\right) \delta 7.53$ and 7.36 (two d, Ar-H, $2 \mathrm{H}, J=8.9$ and $8.6 \mathrm{~Hz}$ ), 6.94-6.71 (m, 13H, Ar-H), 4.75-4.43 (m, 6H, Ar- $\mathrm{CH}_{2}-\mathrm{N}, \mathrm{Cp}-\mathrm{H}$, and $\mathrm{CH}_{2}$ ), 4.35-3.75 (m, $19 \mathrm{H}, \mathrm{Ar}-\mathrm{CH}_{2}-\mathrm{N}, \mathrm{CH}_{2}$, and $\left.\mathrm{Cp}\right), 3.74-3.62\left(\mathrm{~m}, \mathrm{CH}_{2}, 8 \mathrm{H}\right), 3.56-3.15\left(\mathrm{~m}, \mathrm{CH}_{2}, 8 \mathrm{H}\right), 2.29$ and 2.27 (two s, $\mathrm{Ar}-\mathrm{CH}_{3}, 6 \mathrm{H}$ ), 2.16 and 2.14 (two s, N-Ac, 3H) ppm.

3d: ${ }^{1} \mathrm{H}-\mathrm{NMR}\left(\mathrm{CDCl}_{3}, 270 \mathrm{MHz}\right) \delta 7.53$ and 7.36 (two d, Ar-H, $2 \mathrm{H}, J=8.1$ and $8.3 \mathrm{~Hz}$ ), 7.02-6.71 (m, Ar-H, 13H), 4.95-4.91 (m, 2H, Cp), 4.80-4.70 (m, 4H, Ar- $\mathrm{CH}_{2}-\mathrm{N}$ and $\mathrm{Ar}-\mathrm{CH}_{2}-\mathrm{N}$ ), 4.55-4.51 (m, 2H, Cp), 4.38-4.33 (m, 4H, $\mathrm{CH}_{2}$ ), 4.23 (s, 5H. Cp) 3.92-3.68 (m, $\mathrm{CH}_{2}, 8 \mathrm{H}$ ), 3.57-3.07 (m, $\mathrm{CH}_{2}, 8 \mathrm{H}$ ), 2.32 and 2.30 (two s, Ar- $\mathrm{CH}_{3}, 6 \mathrm{H}$ ), 2.23 and 2.20 (two s, N-Ac, $3 \mathrm{H}), 1.89-1.18\left(\mathrm{~m}, \mathrm{CH}_{2}, 20 \mathrm{H}\right) \mathrm{ppm}$.

Model compound 4<smiles>Cc1cc(C)cc(CNCc2ccc(CO)cc2)c1</smiles>

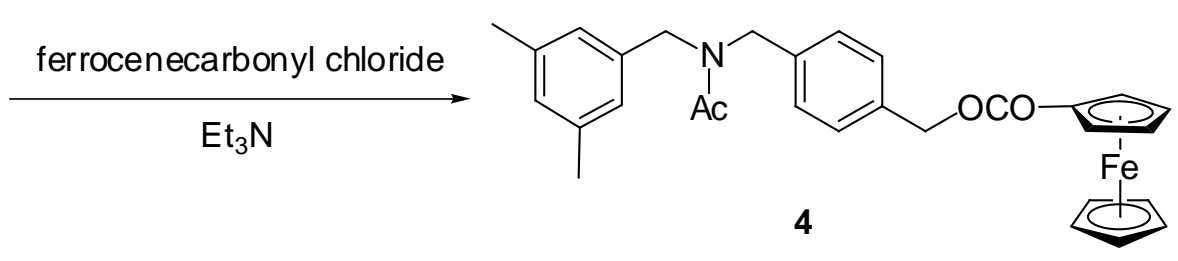

\section{Alcohol 11}

To a solution of aminoalcohol $10(1.07 \mathrm{~g}, 4.19 \mathrm{mmol})$ in dichloromethane $(1.0 \mathrm{~mL})$ were added acetic anhydride $(0.800 \mathrm{~mL}, 8.46 \mathrm{mmol})$ and triethylamine $(1.00 \mathrm{~mL}, 7.17 \mathrm{mmol})$, and the reaction mixture was allowed to stand at room temperature for $40 \mathrm{~h}$. The reaction mixture was washed by water then ammonium chloride solution, dried over magnesium sulfate, and evaporated in vacuo. The crude product was dissolved in dichloromethane (2.0 $\mathrm{mL})$, and methanol $(5 \mathrm{~mL})$ and $5 \%$ sodium hydroxide solution $(10 \mathrm{~mL})$ were added to the 
solution. After the reaction mixture was stirred for $20 \mathrm{~h}$, the organic layer was washed by water then $5 \%$ ammonium chloride solution, dried over magnesium sulfate, and evaporated in vacuo. The crude product was chromatographed (silica gel, eluent: hexane-ethyl acetate) to give $210 \mathrm{mg}(0.679 \mathrm{mmol}, 44 \%)$ of alcohol 11 as a colorless oil.

${ }^{1} \mathrm{H}-\mathrm{NMR}\left(270 \mathrm{MHz}, \mathrm{CDCl}_{3}\right) \delta$ 7.42-7.16 (4H, m), 6.95 and $6.93(1 \mathrm{H}$, two s), 6.84 and 6.77 $(2 \mathrm{H}$, two s), 4.73 and 4.71 ( $2 \mathrm{H}$, two s), 4.61 and 4.54 ( $2 \mathrm{H}$, two s), 4.45 and 4.38 ( $2 \mathrm{H}$, two s), 2.33 and $2.31(6 \mathrm{H}$, two s), $2.23(3 \mathrm{H}, \mathrm{s}) \mathrm{ppm}$.

\section{Model compound 4}

To a solution of alcohol $11(80.0 \mathrm{mg}, 0.270 \mathrm{mmol})$ in dichloromethane $(1.0 \mathrm{~mL})$ were added ferrocenecarbonyl chloride $(67.1 \mathrm{mg}, 0.270 \mathrm{mmol})$ and triethylamine $(42.2 \mu \mathrm{L}, 0.300$ mmol). After the reaction was completed on TLC, the reaction mixture was washed with water then brine, dried over magnesium sulfate, and evaporated in vacuo. The crude product was chromatographed (silica gel, eluent: dichloromethane-methanol) to give $108 \mathrm{mg}(0.212$ mmol, $78 \%$ ) of model compound 4 as an orange viscous oil.

m.p. 102.8-104.0 ${ }^{\circ} \mathrm{C} .{ }^{1} \mathrm{H}-\mathrm{NMR}\left(\mathrm{CDCl}_{3}, 270 \mathrm{MHz}\right) \delta$ 7.50-7.12 (m, Ar-H, 4H), 6.94-6.68 (m, Ar-H, 3H), 5.27 and 5.25 (two s, Ar- $\mathrm{CH}_{2}-\mathrm{O}, 2 \mathrm{H}$ ), 4.84-4.80 (m, Cp, 2H), 4.60-4.49 (m, Ar- $\mathrm{CH}_{2}-\mathrm{N}, 2 \mathrm{H}$ ), 4.41 (s, Cp, 2H), 4.44-4.32 (m, Ar- $\mathrm{CH}_{2}-\mathrm{N}, 2 \mathrm{H}$ ), 2.29 and 2.26 (two s, Ar$\mathrm{CH}_{3}, 6 \mathrm{H}$ ), 2.20 and 2.19 (two s, N-Ac, 3H) ppm.

Ferrocenium cation: General procedure

In a Schlenk tube, a ferrocene drivative $(0.9 \mathrm{mmol})$ was dissolved in freshly distilled ether $(60 \mathrm{~mL})$ under an argon atmosphere. Silver tetrafluoroborate $(210 \mathrm{mg}, 1.08 \mathrm{mmol})$ was added to the solution. After the solvent was removed in vacuo, the flask was flashed by argon, and the residue was dissolved in freshly distilled dichloromethane. The insoluble materials were filtrated off through celite under an argon atmosphere. After the filtrate was concentrated to $2.5 \mathrm{~mL}$, ether $(35 \mathrm{~mL})$ was added. The precipitate was collected by filtration under an argon atmosphere. 


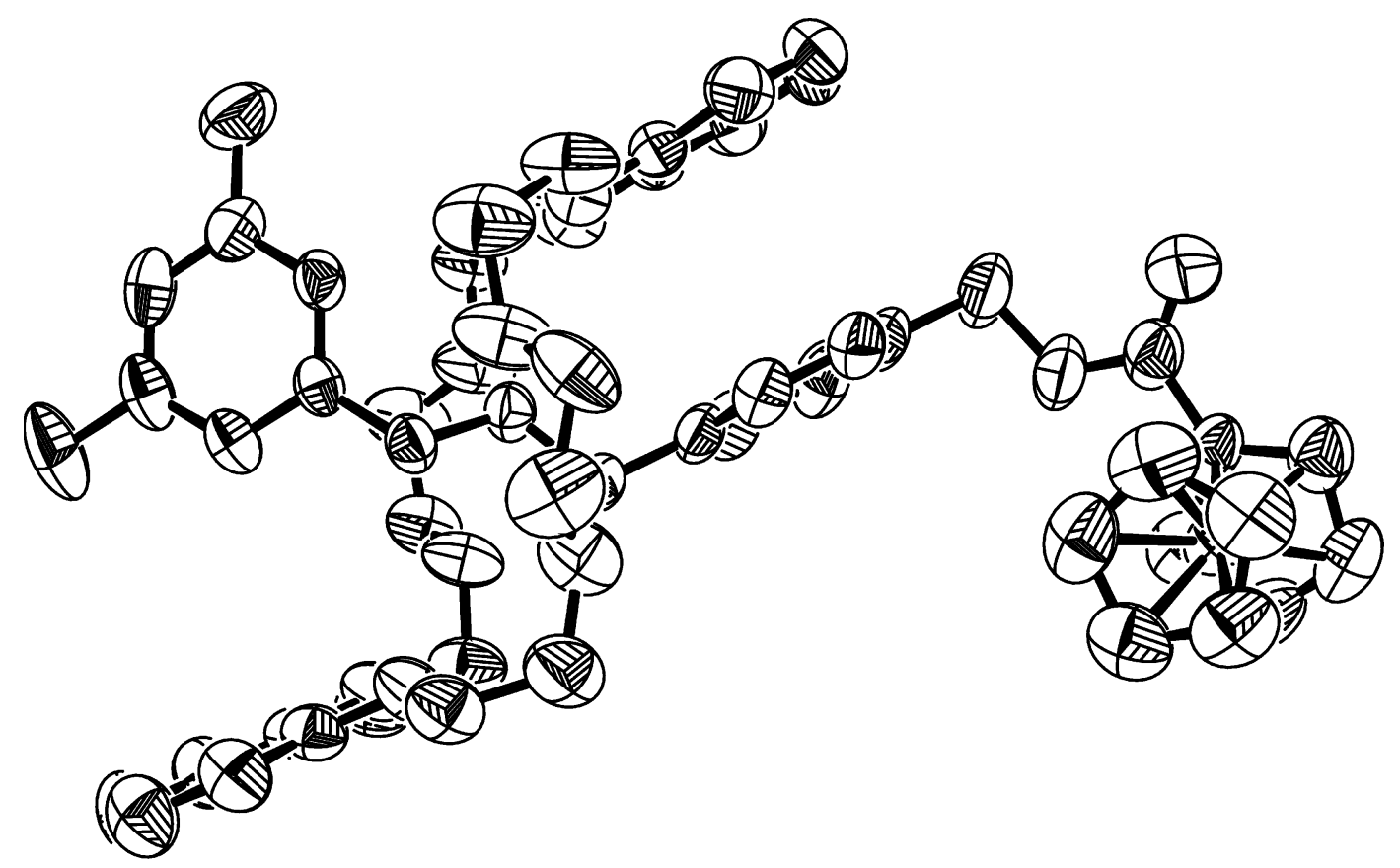

Figure S1. ORTEP drawing of $1 \mathbf{a}^{\cdot} \mathrm{C}_{6} \mathrm{H}_{6}$. Hydrogens, benzene, and $\mathrm{PF}_{6}$ were omitted for clarify.

Crystal data for 1a•benzene: $\mathrm{C}_{58} \mathrm{H}_{68} \mathrm{~F}_{6} \mathrm{FeNO}_{10} \mathrm{P}, \mathrm{FW}=1139.99$, triclinic, $P \mathrm{~T}, a=11.1211(1)$, $b=11.4180(2), c=23.0424(4) \AA, \alpha=101.7369(7), \beta=94.940(1), \gamma=93.0475(9)^{\circ}, V=$ $2846.62(8) \AA^{3}, Z=2, \mu\left(M_{\alpha}\right)=3.694 \mathrm{~cm}^{-1}$, Dcalc $=1.330 \mathrm{~g} / \mathrm{cm}^{3}, R_{1}=0.084, R w=0.176$ (763 variables on $12735, I>2 \sigma(I)), \mathrm{GOF}=1.116$. 


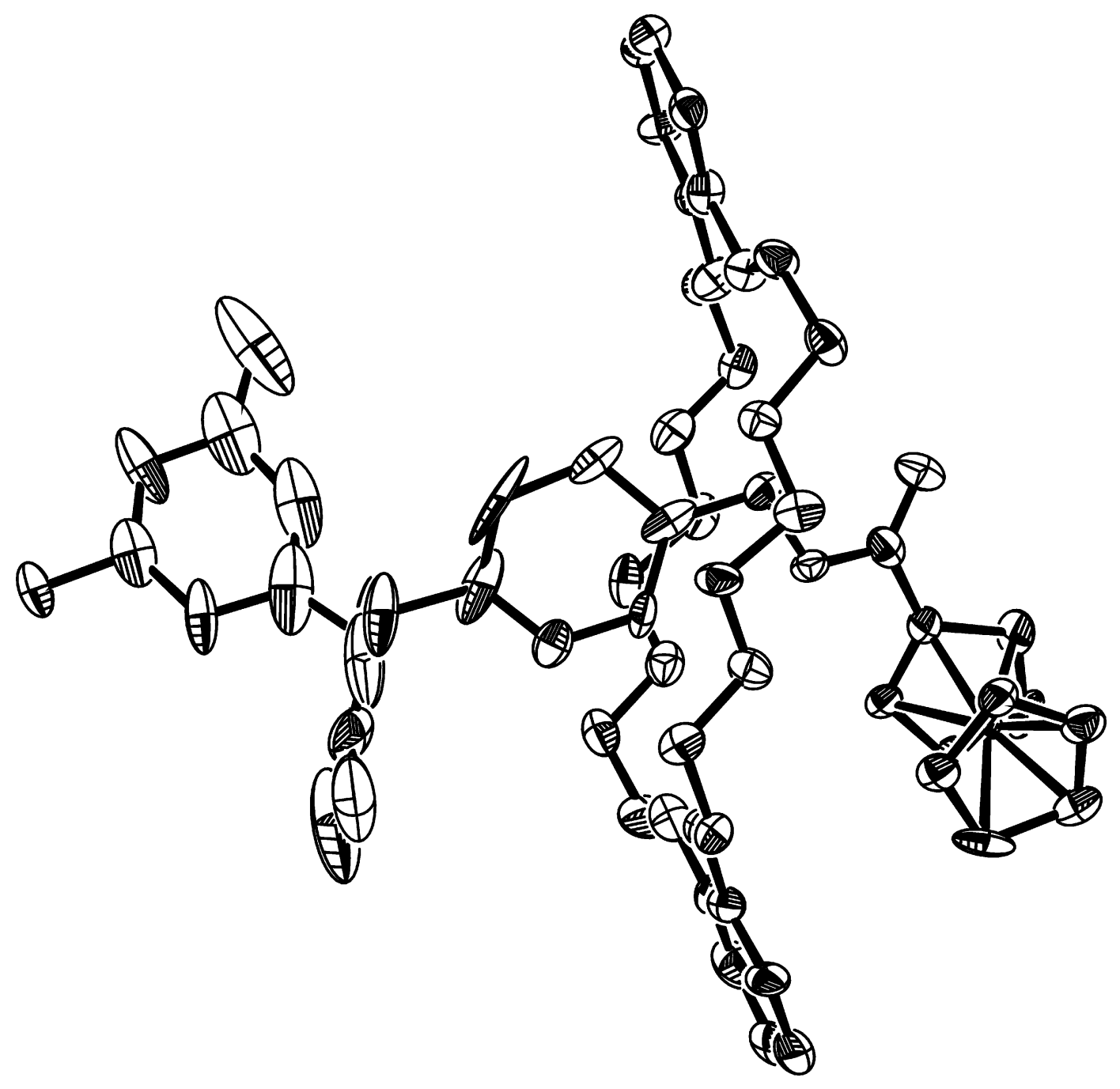

Figure S2. ORTEP drawing of 3a. Hydrogens were omitted for clarify.

Crystal data for 3a: $\mathrm{C}_{54} \mathrm{H}_{63} \mathrm{FeNO}_{11}, \mathrm{FW}=957.94$, triclinic, $P \mathrm{~T}, a=9.8626(1), b=11.6458(1)$, $c=23.4277(4) \AA, \alpha=76.0520(8), \beta=90.3320(5), \gamma=74.071(9)^{\circ}, V=2503.8(1) \AA^{3}, Z=2$, $\mu\left(\mathrm{MoK}_{\alpha}\right)=3.612 \mathrm{~cm}^{-1}$, Dcalc $=1.271 \mathrm{~g} / \mathrm{cm}^{3}, R_{1}=0.133, R w=0.299$ (668 variables on 2789 , $I>2 \sigma(I)), \mathrm{GOF}=1.269$. 
$1 \mathbf{a}$

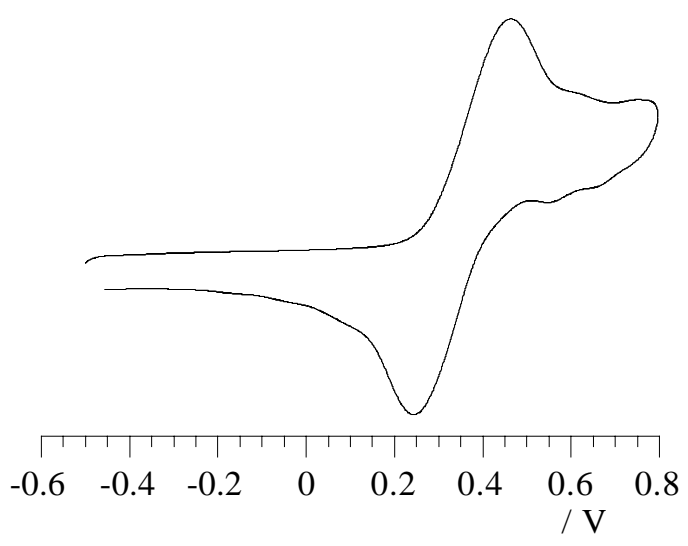

3a

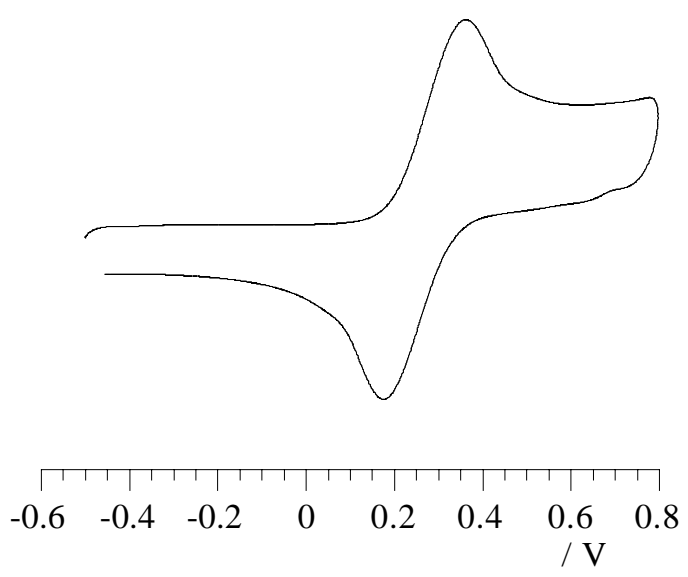

$1 \mathbf{b}$
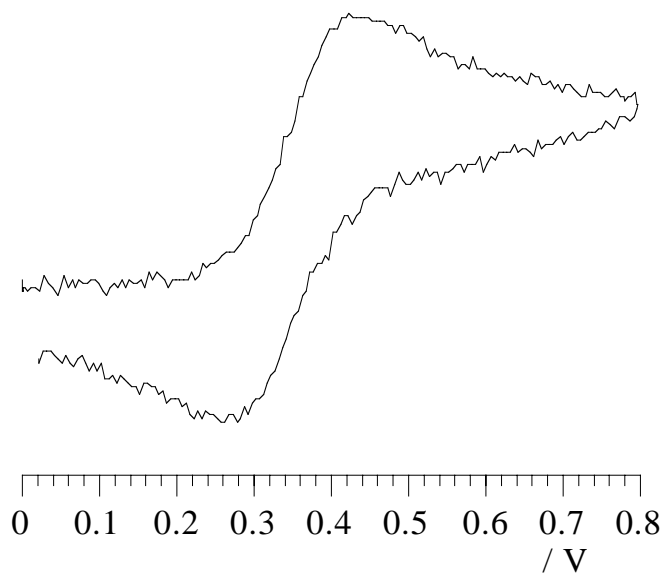

$3 \mathbf{b}$
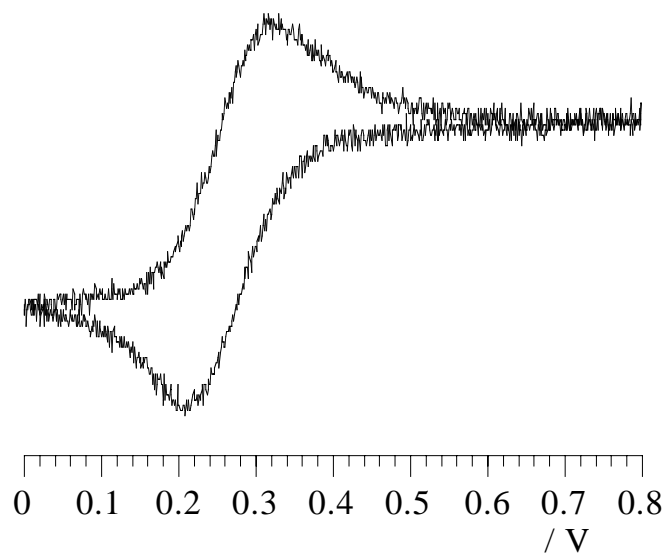

(To be continued)

Figure S3. Cyclic voltammogram of $\mathbf{1}, \mathbf{3}$, and 4 (vs. $\mathrm{Ag} / \mathrm{Ag}^{+}$). Tetrabutylammonium hexafluorophophate $(100 \mathrm{mmol} / \mathrm{L})$ was used as the supporting electrolyte. $[\mathrm{Fc}]=0.5 \mathrm{mmol} / \mathrm{L}$ in acetonitrile. Scan rate, $1 \mathrm{~V} / \mathrm{s}$. 
1c
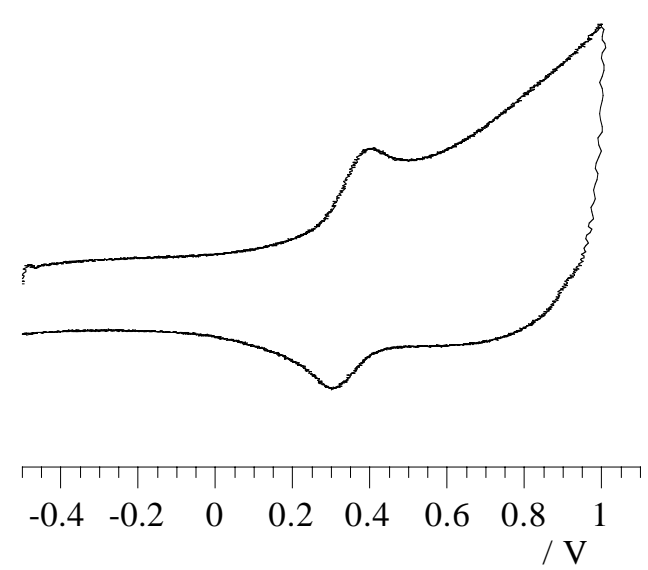

$3 c$
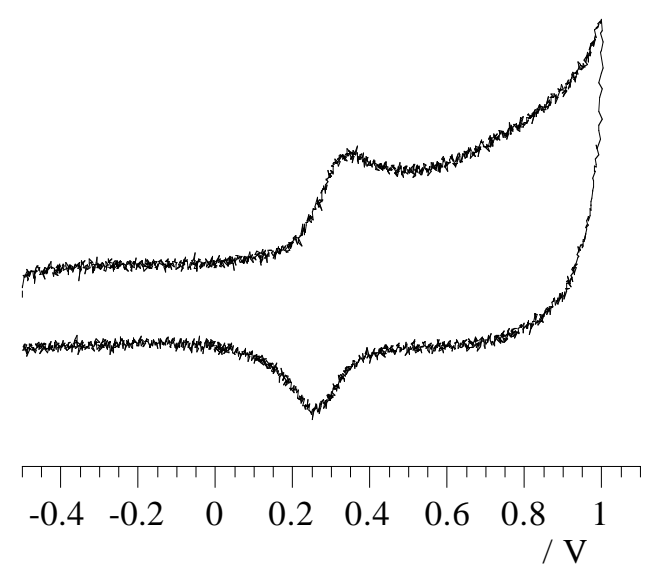

4

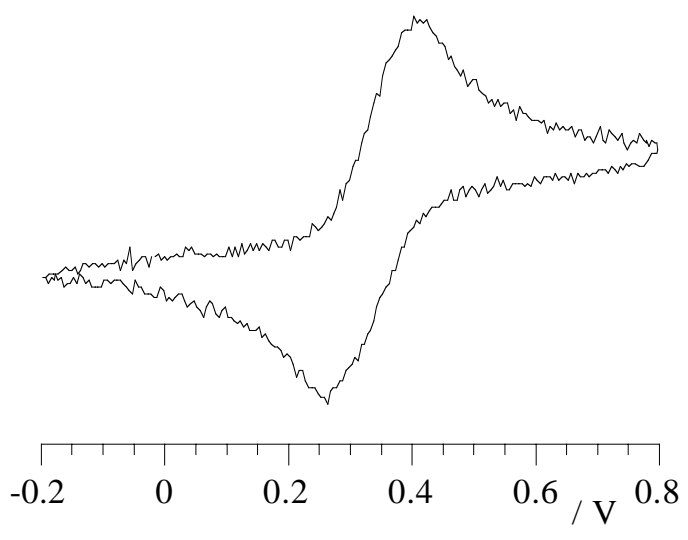

1d
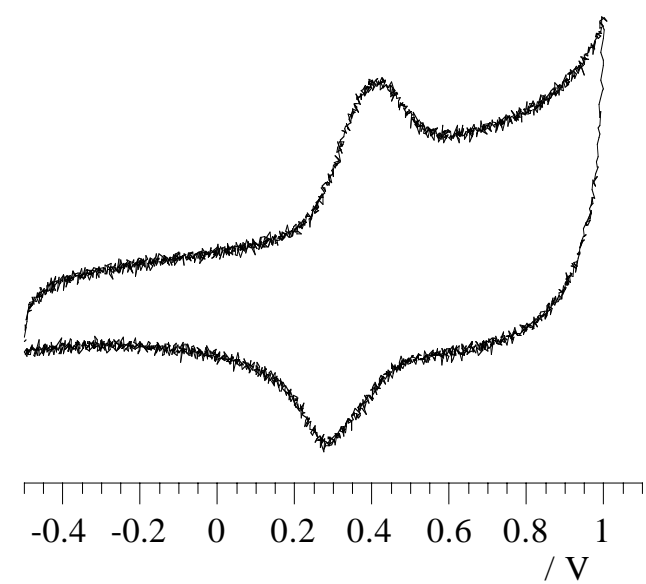

3d
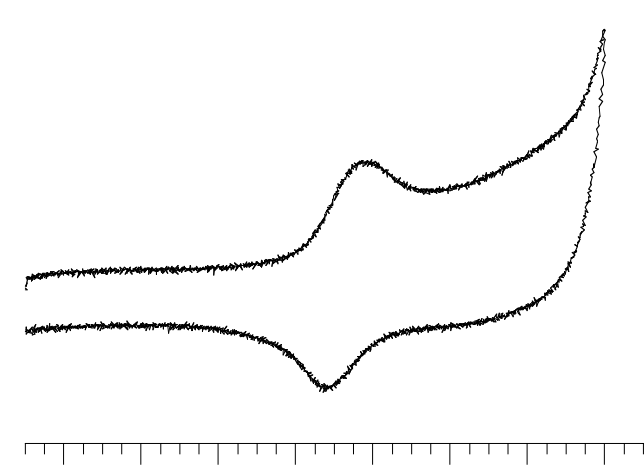

$\begin{array}{cccccccc}-0.4 & -0.2 & 0 & 0.2 & 0.4 & 0.6 & 0.8 & 1 \\ / \mathrm{V}\end{array}$

Figure S3. (Continued) 


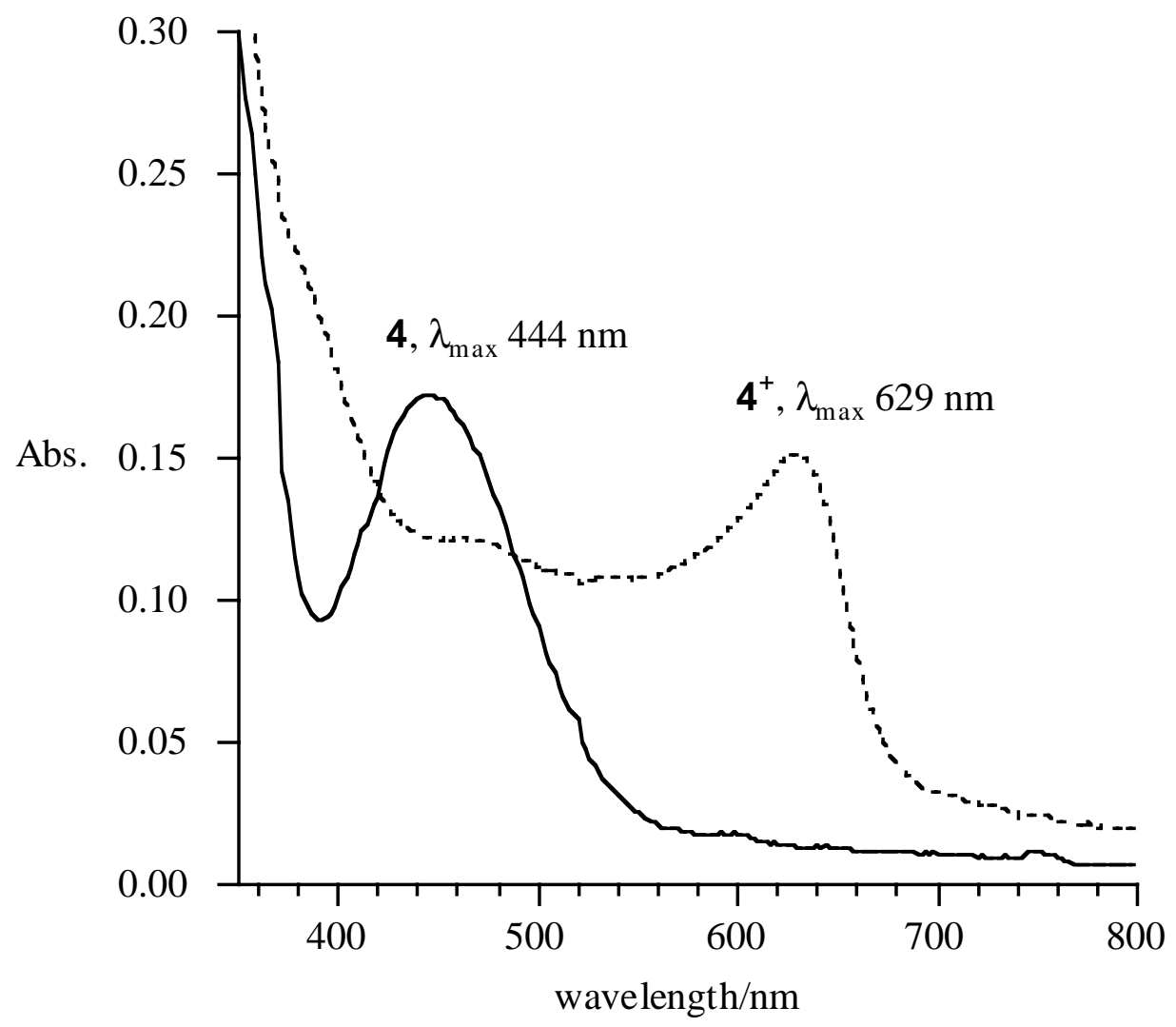

Figure S4. Electronic spectra of $\mathbf{4 a}$ (solid line) and $\mathbf{4 a}^{+}$(dashed line) in $\mathrm{CH}_{2} \mathrm{Cl}_{2}$ at room temperature. 